body weight) and one hour later with $0.2 \mathrm{ml}$. of $0.5 \%$ quinine hydrochloride solution.

2. Quinine group. The birds were injected daily with $0.2 \mathrm{ml}$. of physiological saline solution and one hour later with $0.2 \mathrm{ml}$. of $0.5 \%$ quinine hydrochloride.

In both groups the injections were given intramuscularly and repeated for four consecutive days.

3. Untreated control group.

From the fourth day of inoculation parasites were examined almost daily microscopically by Giemsa's method. The intensity of their development was estimated by counting them in a given number of fields.

On an average, the degree of development of parasites on the 12th day in the quinine-thiosulphate group corresponded approximately to that of the 9th day of the quinine group, and it can be said that in the experimental conditions thiosulphate brought about an inhibition equivalent to about three days. This result may be attributable, apart from the three factors already mentioned, to some degenerative changes in the protoplasmic constitution of the microbes caused by direct action of thiosulphate upon itKritschewski's thiosulphate phenomenon. ${ }^{4}$ Further, it can be expected that quinine may be replaced by mepacrin, or any other new antimalarial agent.

Systematic clinical examinations of the combined treatment suggested above have not yet been undertaken in Japan, but experience has shown us that the most adequate route of administration of thiosulphate is the intravenous injection.

In conclusion we hope that the field of the combined application of thiosulphate as a common, non-toxic activator of chemotherapy need not be restricted to malaria, but will find a widespread extension irrespective of the nature of the chemotherapeutic agent-e.g., sulphanilamide, etc. Out of three cases of kala-azar, esistant to antimony preparations, successful results were obtained promptly through the combined thiosulphate treatment.-We are, etc.,

\section{J. HIRADE}

Tokyo, Japan.

\section{Y. YASLI}

\section{REFERENCES}

1 J. trop. Med. Hyg., 1946, 49, 63.

2 Hirade, J., and Yasui, Y., Medical Review (Igaku-sóran), 1945, No. 6. I Z. ImmunForsch., 1931, 73, 303.

\section{Poliomyelitis and Lymphocytic Meningitis}

SIR,-When presenting his neurological experiences in the Middle East and India at the Royal Society of Medicine, Dr. Douglas McAlpine ${ }^{1}$ in his discussion on acute benign lymphocytic meningitis stated that "neutralization tests in some of these proved that the majority were not due to the virus of acute choreomeningitis." He then went on to say that "in 1941 abortive cases of poliomyelitis were reported by Major Caughey, N.Z.A.M.C., thus making the sorting of these cases of lymphocytic meningitis more difficult."

The facts are as follows. Between November, 1940, and July, 1941, an epidemic of poliomyelitis occurred among New Zealand troops in the Middle East. This has been recorded in the Medical Journal of Australia by myself and Dr. Porteous. ${ }^{2}$ Out of 40 cases 21 were the pre-paralytic type, with changes in the spinal fluid but no paresis. At the time Dr. McAlpine stated in a report that these cases were not cases of poliomyelitis, although they had come from the same division, the same units, and occurred at the same time as the 19 paralytic cases.

In the Journal of Dec. 27, 1947 (p. 1019), in a paper on acute poliomyelitis, Dr. McAlpine and others again make reference to acute benign lymphocytic meningitis when discussing the differential diagnosis. When speaking of acute choreomeningitis they state that they "are not convinced that up to the present this condition has been at all common in this country [U.K.], although it should be added that many cases werc seen in Service personnel in the Middle East during the late war." There is a marked discrepancy between this latter statement and that Dr. McAlpine made in 1945 at the Royal Society of Medicine. As far as I was aware on no occasion was it established by neutralization tests that acute choreomeningitis occurred in the Middle East among Service personnel, but I am open to correction on this point.

Finally, Dr. McAlpine and others state in the summing-up that in England "the virus of acute poliomyelitis should now be regarded as the usual cause of a benign type of lymphocytic meningitis." Hence they will allow of the diagnosis of preparalytic cases of poliomyelitis in the United Kingdom, but
Dr. McAlpine persists in his denial of such cases in the epidemic of poliomyelitis in the Middle East. He cannot have it both ways.

I am entirely in agreement that during an epidemic of poliomyelitis by far the most likely cause of cases of acute lymphocytic meningitis is poliomyelitis, but the exact diagnosis can only be made by serological tests and animal tests. Until such are more readily available, during an epidemic of poliomyelitis cases of acute lymphocytic meningitis must be regarded as being due to poliomyelitis.-I am, etc.,

Auckland. New Zealand.

\section{J. E. Caughey.}

REFERENCES

1 Proc. R. Soc. Med., 1946, 39, 169.

2 Med. J. Aust., 1946, 1, 5.

\section{Fear and Pain in Childbirth}

SiR,-Dr. J. Donaldson Craig (April 10, p. 706) makes the point that the pain of labour may be modified, if not entirely eliminated, "by the degree of confidence reposed in medical attendants." A very great deal has been written about the physical pain of labour, but little attention has been paid to what Head describes as "the diminution of general resistance to painful impressions" by "debilitating psychological states, such as anxiety or emotional shock." If certain obstetricians are able to conduct relatively painless labours without anaesthesia, surely it is by the elimination of fear. Is it not time that we devoted more attention to this aspect of the matter?

It is difficult for anyone long familiar with anatomy and accustomed to the facts relating to the physiology of labour to enter imaginatively into the state of chaotic ignorance in which many women are still allowed to approach their first confinement. IIIequipped with an assortment of half-digested facts about her body acquired from popular medical literature and first-aid classes, the young primigravida waits in the clinic queue next to a loquacious mother of five. She may be encouraged by the resulting confidences, as Dr. H. Thistlethwaite (Feb. 14, p. 318) optimistically suggests, but unfortunately we know that she is more likely to gather a terrifying impression of "instruments" and "stitches." Her own idea of the birth canal must inevitably be that it is small, since her experience of it is limited to coitus and the insertion of vaginal tampons. How can so small an aperture allow the escape of a baby without some ghastly destructive process? As a rule this fear and its associated images are suppressed, and are only shown by an inability to relax during examinations. A simple but graphic explanation about the size of the bony pelvis and the softening processes in the concertina-like vagina will give immediate and lasting relief. If our clinics are too crowded to allow time to probe for these fears, information should be made available to mothers through simple lectures or discussion groups.

The primigravida frequently comes to her confinement in a state of apprehension. In many maternity departments she is admitted in a glare of lights on to a high white bed with a good view of the instrument cupboard. A sterilizer may be boiling up the forceps, presently to be lifted out in front of her, for Mrs. Jones moaning audibly next door. In this year of grace there are still communal labour wards, and she mav find herself in the company of one or more pain-weary women more advanced in labour than herself. Confidences are soon exchanged, and they are not such as to reassure. Someone in a white coat appears and examines her. Notes are written and drugs ordered, but remarks to the patient are confined to kindly meaningless phrases which give nothing away and therefore do not allay her fears.

Kindness, though it abounds, is unfortunately not enough. We are all straining to be kind, but custom tends to dull our perception of the inner significance of birth as it is experienced by the mother. It is easy to forget the fear of the unknown and the sense of mystery and miracle which preoccupy her mind while we are debating some obstetric problem. We need to be more sensitively aware of the emotional side of the patient's experience if we are to avoid all those petty unconscious cruelties to which we subject her by our failure of imagination. By eliminating these we may do much to remove the part of pain which is made unendurable by fear, and the fear which may in itself be worse than pain.-I am, etc.,

London, W.11.

MARY E. EgERTON 